\title{
One-session bilateral sequential whole lung lavage (OSBSWLL) for the management of pulmonary alveolar proteinosis
}

\author{
Javier Diaz-Mendoza ${ }^{1,2^{*}}$, Eduardo Celis Valdiviezo ${ }^{3,4}$, Niral M. Patel ${ }^{5}$ and Michael J. Simoff ${ }^{1,2}$
}

\begin{abstract}
Background: Whole Lung Lavage (WLL) has been an important part in the management of Pulmonary Alveolar Proteinosis (PAP) since it improves radiologic and clinical parameters. Bilateral WLL is usually performed in two sessions on different days. Few case reports have described one-session bilateral sequential lung lavage (OSBSWLL), and none have described ambulatory management (same-day discharge).

Methods: Demographic characteristics, physiologic parameters, procedure details and outcomes were retrospectively collected on consecutive patients who underwent OSBSWLL for PAP following an ambulatory protocol stablished in our institution.

Results: A total of 13 patients underwent 30 OSBSWLL (61.5\% male; mean age 40). The mean SpO2 was 90\% (IQR 9) and $94 \%$ (IQR 6), before and after OSBSWLL respectively. In 63.3\% of cases, patients were discharged home the same day of procedure. Only in two cases (6.6\%), patients required post-procedure prolonged mechanical ventilation ( $>4 \mathrm{~h}$ ) due to persistent hypoxia.
\end{abstract}

Conclusions: OSBSWLL can be performed with same-day discharge.

\section{Introduction}

Pulmonary Alveolar Proteinosis (PAP) was recognized by Rosen in 1958 [1]. It is a rare entity with a prevalence between 3.2 to 6.7 cases per million [2,3]. More than $90 \%$ of PAP cases occur as primary acquired disorders of unknown etiology [1].

The diagnosis of PAP is based on the clinical suspicion and radiologic findings (bilateral and symmetrical confluent airspace disease [4], and/or "crazy paving" pattern seen on computed tomography [5]. The bronchoalveolar lavage effluent has a turbid and milky appearance due to the presence of foamy macrophages or monocyte-like

\footnotetext{
*Correspondence: Jdiaz1@hfhs.org

${ }^{1}$ Interventional Pulmonology, Division of Pulmonary and Critical Care Medicine, Henry Ford Hospital, K-17, 2799 W Grand Blvd, Detroit, Ml 48202, USA

Full list of author information is available at the end of the article
}

alveolar macrophages with an increased number of lymphocytes. These changes are associated with a large periodic acid-Shift (PAS) positive acellular background of diffuse eosinophilic bodies $[4,6]$, which is diagnostic of PAP. The presence of lamellar bodies under electron microscope examination can be used to confirm the diagnosis. These abnormalities translate into an enormous accumulation of surfactant lipoprotein in pulmonary alveoli leading to the clinical findings of increase alveolar-arterial oxygen gradient and hypoxemia.

Whole Lung Lavage (WLL), first described by RamirezRivera in 1965 [7], is currently the first line therapy for PAP. This procedure is performed to remove the excess surfactant proteins and lipids from the alveolar spaces in order to improve gas exchange. Most published case series, as well as a recent global survey, have shown that most centers perform WLL on two separate sessions [8]. In our institution, we have been performing bilateral, 
sequential WLL on one session (OSBSWLL) since 1994, although inconsistently initially, it became more consistent over the past 15 years. We believe that OSBSWLL is safe to perform, comfortable for the patient, and could reduce costs of a second procedural session. Per our knowledge, this is the largest case series of OSBSWLL reported.

\section{Methods}

We retrospectively reviewed the medical records of patients who underwent WLL due to PAP from 1994 until 2013 at Henry Ford Hospital. Our Institutional Review Committee (IRB) had approved this study (IRB-9021) in accordance with the relevant guidelines and regulations. A total of 25 consecutive patients who underwent WLL were identified. All patients had biopsy proven PAP. 13 patients underwent a total of 30 OSBSWLL. Data regarding demographics, clinical presentation, procedure, and complications were collected (Table 1).

\section{Procedure/technique Before lavage}

All OSBSWLL are performed in the Operating Room (OR) under general anesthesia. The team involved in the procedure include: an interventional pulmonologist, an anesthesiologist, respiratory therapists, and experienced operating room assistants. In the operating room, general anesthesia and muscle paralysis are induced by the anesthesiologist. The patient is placed in a supine position and intubated with a $39 \mathrm{Fr}$ doublelumen endotracheal tube (dETT) (or smaller depending on the size of patient). After the position of the
dETT in the airways is confirmed by flexible bronchoscopy (pediatric scope), the dETT is secured in place. A "leak test" is then performed: a single lumen endotracheal tube is attached to a connector allowing it to be secured to the dETT, with the tip placed in a bottle of saline, which will hence be referred to as the leak test kit (LTK). Initially one side of the dETT is connected to the LTK and the other to the ventilator. Airway pressures are increased by the ventilator and held sequentially at 20,30 and $40 \mathrm{~cm} \mathrm{H} 2 \mathrm{O}$. The airway pressures are then fluctuated between 40 and $50 \mathrm{~cm} \mathrm{H} 2 \mathrm{O}$ looking for bubbling in the saline from the end of the LTK. If no bubbling is observed, the process is repeated on the opposite side of the dETT. If both "leak tests" are negative, we proceed to stabilize the head of patient and tubing system by using towels, blankets and padding to avoid pressure ulcers on the patient's face and skin (Fig. 1). The flexible bronchoscope is then used to confirm positioning one more time.

The patient is then oxygenated with $100 \% \mathrm{FiO} 2$ to both lungs for $10 \mathrm{~min}$. After the first lung to be lavaged is determined (the lung with the greater disease burden by imaging), we proceed to the "degassing maneuver". For this, the corresponding arm of the dETT is closed using a curved Kelly clamp. The dETT remains clamped for 5 min to allow degassing of the lung. [9] The customized tubing system to be used for the lavage (Fig. 2) is prepared, ensuring that it is air-free. It is then connected to the appropriate arm of the dETT. Multiple three-liter bags of normal saline had been previously warmed to a temperature of $37{ }^{\circ} \mathrm{C}$. The bags are then hung $40 \mathrm{~cm}$ above the level of the heart.

Table 1 General demographics

\begin{tabular}{|c|c|c|c|c|c|c|}
\hline Patient & Age & Gender & Race & Diagnosis & Year of diagnosis & $\begin{array}{l}\text { Years } \\
\text { to first } \\
\text { OSBSWLL }\end{array}$ \\
\hline 1 & 53 & M & AA & BAL TBBX & 1996 & 4 \\
\hline 2 & 64 & M & C & BAL TBBX & 1996 & $<1$ \\
\hline 3 & 29 & $\mathrm{~F}$ & AA & BAL TBBX & 2000 & $<1$ \\
\hline 4 & 35 & M & $\mathrm{H}$ & OLBX & 2000 & $<1$ \\
\hline 5 & 29 & $F$ & C & OLBX & 2012 & $<1$ \\
\hline 6 & 50 & M & C & OLBX & 1997 & 1 \\
\hline 7 & 16 & M & C & BAL TBBX & 2000 & $<1$ \\
\hline 8 & 44 & M & C & OLBX & 2007 & $<1$ \\
\hline 9 & 46 & $\mathrm{~F}$ & C & OLBX & 2007 & 2 \\
\hline 10 & 46 & $\mathrm{~F}$ & AA & OLBX & 2009 & 2 \\
\hline 11 & 32 & M & $C$ & OLBX & 1996 & $<1$ \\
\hline 12 & 40 & $F$ & $C$ & BAL TBBX & 1995 & $<1$ \\
\hline 13 & 44 & M & C & OLBX & 1994 & $<1$ \\
\hline
\end{tabular}




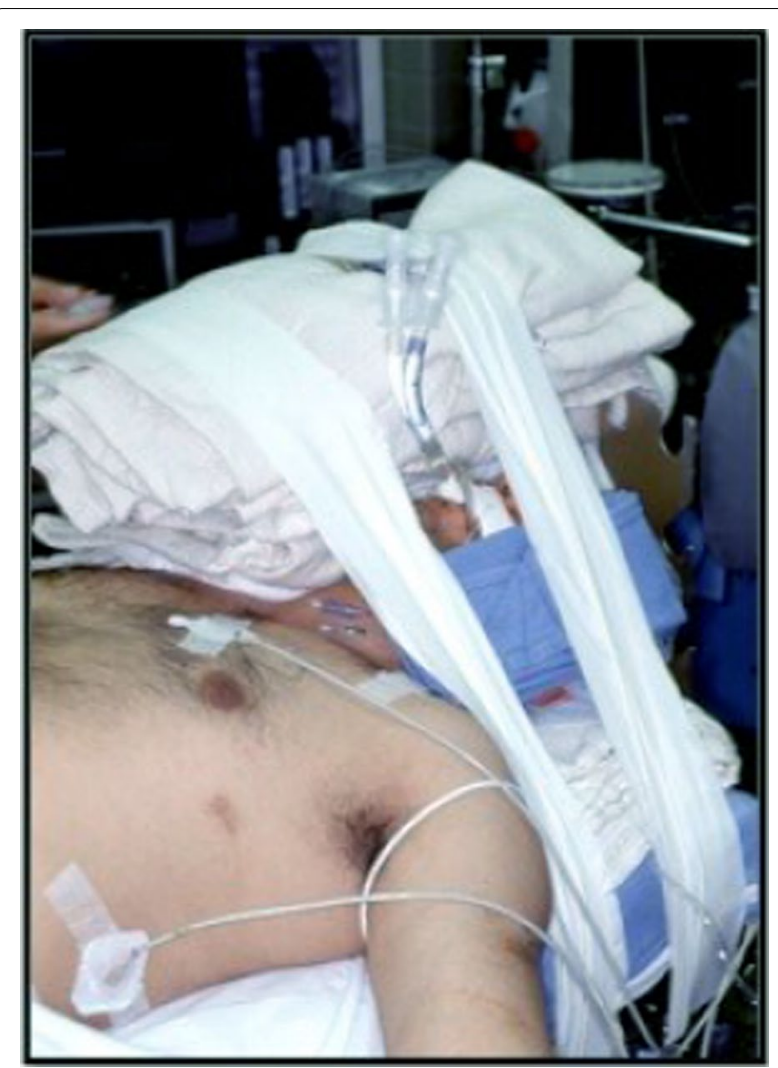

Fig. 1 Patient intubated with a double lumen endotracheal tube supported by towels achieving airway stabilization and protection of patient soft tissue areas over the neck, face and head

\section{Lavage}

We start the lavage by instilling two liters of the warmed normal saline, by gravity, into the lung through the tubing system. Cardiopulmonary physical therapists provide manual percussion of the chest throughout the procedure in order to achieve better mobilization and subsequently improved removal of particulate matter and instilled fluid. After the first two liters are instilled into the lung, the inflow portion of the tubing circuit is closed and the effluent from the lung is drained by gravity into a suction canister which is calibrated by milliliters. Initially, one liter of effluent is drained and one liter of saline remains in the lung to maintain patency of the lung. The process of instillation and drainage is repeated using one-liter aliquots for both the in and out portions of the procedure. Visible clearing of the effluent is used as the marker to stop the lavage. Effluent clearing usually requires approximately $16 \mathrm{~L}$ of fluid per lung. When the effluent has cleared enough (Fig. 3), the final drainage will include the one liter of initially instilled into the lung.

The patient is then reassessed to decide if the procedure for the second lung can safely be completed. Assessment of vital signs, including pulsoximetry, as well as bronchoscopic confirmation of the dETT are done before moving on to the second lung. Then we proceed to perform the "degassing maneuver" on the second lung (as described above).

The amount of fluid instilled and removed is carefully ascertained at the completion of each lung and then for a total for the procedure. The "final balance of fluid" (total amount of fluid instilled into the lungs minus amount of fluid recovered from the lungs) is recorded.

\section{After lavage}

At procedure terminus, the double-lumen tube is removed and a \#8 single-lumen ETT is placed (depending on the size of patient). The patient is maintained on mechanical ventilation for $4 \mathrm{~h}$ following the procedure. For the first $2 \mathrm{~h}$ on an $\mathrm{FiO} 2$ of $100 \%$ and for the second $2 \mathrm{~h}$ weaned to $30 \%$. If patients are assessed to be clinically doing well with adequate $\mathrm{SaO} 2$ they are discharged home the same day of the procedure. Patients admitted to the hospital overnight will be weaned to room air prior to discharge. All patients are scheduled for outpatient follow up within 1 to 2 weeks.

\section{Results}

Thirteen patients underwent a total of 30 OSBSWLL (mean of 2.3 OSBSWLL per patient, IQR 1). There were two other procedures scheduled initially as OSBSWLL, but they only completed a single WLL due to complications. These 2 cases were not included on the 30 OSBSWLL (Table 2).

Eight patients were male (61.5\%). Nine were Caucasians (69.2\%). PAP was diagnosed with Open Lung Biopsy (OLBX) in 8 patients (61.5\%). The time from diagnosis of PAP to first OSBSWLL ranged from less than one year to 4 years (mean 1.38; IQR 0.5) (Table 1 ).

None of the patients were on mechanical ventilation before OSBSWLL, and only two were inpatients before the procedure (6.6\%).

The time used for OSBSWLL was found in 17 procedures. The time varied between 135 and 274 min (mean of $208.1 \mathrm{~min}$, IQR 42). The total amount of normal saline (in Liters) used per OSBSWLL ranged between 14.5 and 50.9 (mean of 31.1, IQR 15.3) with a range between 6 to 29.3 per lung (mean of $15.5 \mathrm{~L}$ ). The final balance of fluid at the end of the procedure ranged between 0 and $2500 \mathrm{ml}$ (mean of $781.8 \mathrm{ml}$, IQR 790).

Peripheral capillary oxygen saturation (SpO2) values on room air were found in 21 cases (15 pre-procedure, 19 post-procedure, and 13 in both). The mean SpO2 pre-procedure was $90 \%$ (IQR 9). The mean SpO2 in the immediate post-procedure was $94.8 \%$ (IQR 6). Among the cases with both values, the mean variation between the pre and post-procedure $\mathrm{SpO} 2$ was $+5.3 \%$ (IQR 9.5). 

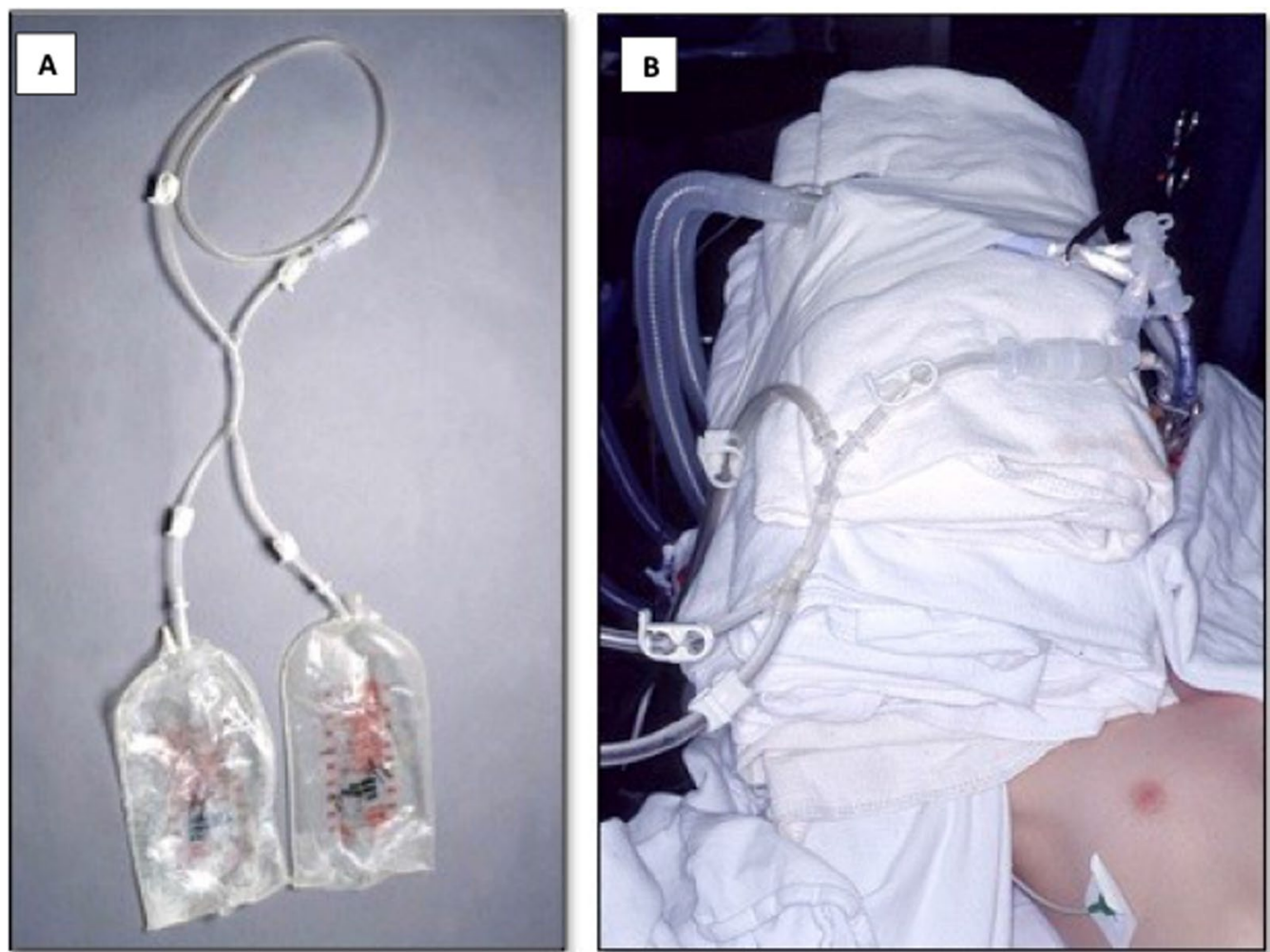

Fig. 2 A Tubing setting. B Tubing setting seen on patient during procedure

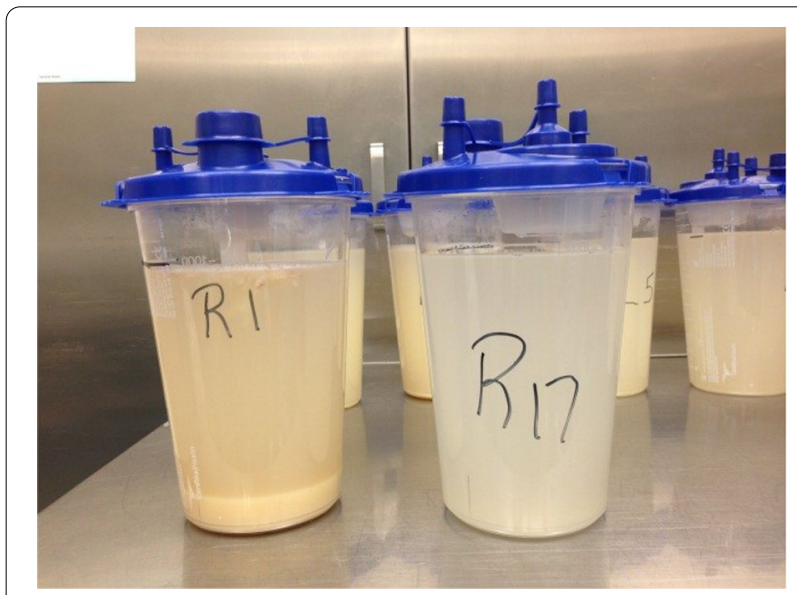

Fig. 3 Example of visual assessment of returned lavage. R1: first returned lavage, right lung. R17: 17th returned lavage, right lung
Eleven cases of OSBSWLL (36.6\%) required postprocedure admission to the hospital. The most common cause for admission was observation for dyspnea (Table 3). The mean length of admission was 2.3 days (IQR 1). Only in 3 cases of OSBSWLL patients required admission to the Intensive Care Unit (two cases with 1 day and one case with 7 days) (Table 3).

In 19 cases of OSBSWLL (63.3\%), the patients were discharged home the same day of procedure. About 1-year survival, 24 cases $(80 \%)$ of OSBSWLL were alive after 1 year, 1 had died and 5 were unknown.

\section{Discussion}

It is known that WLL is the standard treatment for PAP, since it has a great efficacy to improve symptoms as well as other functional and radiological parameters [1]. There

Table 2 Procedures scheduled as OSBSWLL, but only completed lavage of one lung

\begin{tabular}{|c|c|c|c|c|}
\hline Patient & Reason for stopping procedure & Admission to hospital & ICU days & $\begin{array}{l}\text { Total } \\
\text { hospital } \\
\text { days }\end{array}$ \\
\hline 10 & Pneumothorax, needed chest tube placement & yes & 3 & 5 \\
\hline 12 & Hemodynamically instability, that resolved spontaneously & no & NA & NA \\
\hline
\end{tabular}


Table 3 Procedure, indication and hospital admission per patient

\begin{tabular}{|c|c|c|c|c|c|c|c|c|c|}
\hline Patient & $\begin{array}{l}\text { Reason for } \\
\text { OSBSWLL }\end{array}$ & $\begin{array}{l}\text { SpO2 pre/post } \\
\text { lavage at rest on } \\
\text { Room air }(\%)\end{array}$ & $\begin{array}{l}\text { NS used } \\
\text { Left lung } \\
\text { (L) }\end{array}$ & $\begin{array}{l}\text { NS used } \\
\text { Right lung } \\
\text { (L) }\end{array}$ & $\begin{array}{l}\text { Residual } \\
\text { volume } \\
(\mathrm{mL})\end{array}$ & $\begin{array}{l}\text { Length of } \\
\text { procedure } \\
\text { (min) }\end{array}$ & Reason for admission & ICU days & $\begin{array}{l}\text { Total } \\
\text { hospital } \\
\text { days }\end{array}$ \\
\hline 1 & WD, WI & $-/-$ & 17 & 15 & 300 & - & NA & 0 & 0 \\
\hline 2 & WD, WI & $-/ 80$ & 8 & 6.5 & 2500 & - & Hypoxia, pneumonia & 7 & 13 \\
\hline 3 & WD, WI & $-/-$ & 17 & 15 & 415 & - & Observation (dyspnea) & 0 & 3 \\
\hline 3 & $W D, W l$ & $-/-$ & 27 & 18 & 0 & - & NA & 0 & 0 \\
\hline 4 & $W D, W I$ & $-/ 84$ & 17 & 6 & 1000 & - & Hypoxia & 1 & 1 \\
\hline 4 & WD, WI & $-/-$ & 10.2 & 9.7 & 1020 & - & Observation (dyspnea) & 0 & 1 \\
\hline 5 & WD, WI & $95 / 98$ & 29.3 & 21.6 & 1025 & 256 & Tachycardia & 0 & 1 \\
\hline 5 & WD, WI & $92 / 92$ & 21 & 18 & 250 & 219 & Observation (dyspnea) & 0 & 1 \\
\hline 6 & WD, WI & $-1-$ & 9 & 15 & 1000 & - & NA & 0 & 0 \\
\hline 7 & $W D, W l$ & $-/-$ & 18 & 15 & 320 & - & NA & 0 & 0 \\
\hline 8 & WD, WI & $96 / 90$ & 21 & 21 & 325 & 135 & Observation (dyspnea) & 0 & 1 \\
\hline 8 & WD, WI & $94 / 98$ & 15 & 21 & 1090 & 207 & Observation (dyspnea) & 1 & 1 \\
\hline 8 & WD, WI & $94 / 96$ & 18 & 21 & 2100 & 206 & Observation (dyspnea) & 0 & 1 \\
\hline 9 & WD, WI & $95 / 94$ & 19.1 & 24 & 80 & 234 & NA & 0 & 0 \\
\hline 9 & WD, WI & $92 / 97$ & 17.8 & 30 & 200 & 268 & NA & 0 & 0 \\
\hline 10 & WD, WI & $91 / 97$ & 21 & 28 & 1925 & 274 & Observation (dyspnea) & 0 & 2 \\
\hline 11 & WD, WI & $-/-$ & 21 & 17.5 & 200 & - & Observation (dyspnea) & 0 & 1 \\
\hline 11 & $W D, W I$ & $-/-$ & 12 & 12 & 500 & - & NA & 0 & 0 \\
\hline 12 & WD, WI & $-/ 100$ & 12 & 13 & 830 & 195 & NA & 0 & 0 \\
\hline 12 & WD, WI & $-/ 98$ & 15 & 15 & 1050 & 170 & NA & 0 & 0 \\
\hline 12 & WD, WI & $89 / 97$ & 9 & 12 & 1350 & 200 & NA & 0 & 0 \\
\hline 12 & WD, Wl & $-/ 98$ & 11 & 12.7 & 0 & 185 & NA & 0 & 0 \\
\hline 12 & WD, WI & $84 / 100$ & 12 & 18 & 550 & 170 & NA & 0 & 0 \\
\hline 12 & WD, WI & $85 / 100$ & 12 & 15 & 500 & 220 & NA & 0 & 0 \\
\hline 12 & WD, WI & $83 / 96$ & 12 & 15 & 500 & 185 & NA & 0 & 0 \\
\hline 12 & WD, WI & $86 / 90$ & 15 & 15 & 0 & 200 & NA & 0 & 0 \\
\hline 12 & WD, WI & $84 /-$ & 15 & 15 & 850 & - & NA & 0 & 0 \\
\hline 12 & WD, WI & $-/-$ & 9 & 12 & 1300 & - & NA & 0 & 0 \\
\hline 12 & WD, WI & 90/- & 9 & 9 & 1100 & - & NA & 0 & 0 \\
\hline 13 & WD, WI & $-/ 97$ & 9 & 9 & 1175 & 215 & NA & 0 & 0 \\
\hline
\end{tabular}

WD: worsening dyspnea (subjective to patient); WI: worsening chest X-Ray or chest computed tomography images; NA: non applicable; NS: normal saline; L: liters; mL: milliliters; -: no value found

is still a lack of standardization of the indications for the procedure, the procedure itself and the outcomes [8].

Traditionally, most case series of PAP have mentioned the use of WLL in two separate sessions (one lung per session) $[3,4,6,8]$. A global survey on WLL done in 20 centers in 14 countries revealed that most centers perform WLL in consecutive sessions (one lung per session), with an interval of 1 to 2 weeks between procedures [8]. No specific data has been published about OSBSWLL thus far.

In this case-series, we present our experience with OSBSWLL as part of the treatment for PAP. We believe this is the largest case-series report for OSBSWLL in the literature.
The most common indications to perform WLL described are: lung function decline, change in $\mathrm{PaO} 2$ or $\mathrm{SpO} 2$, worsening chest imaging (chest X-ray or computed tomography) and worsening dyspnea $[3,4,8]$. In our case-series, all the patients had dyspnea and worsening chest images before undergoing OSBSWLL. The mean $\mathrm{SpO} 2$ value before procedure was also low (90\%).

The amount of fluid used per lung in our series has a great variation (6 to 29.3 Liters), which is comparable to other series (range between 1 and 40 Liters) [1, 8]. In our institution the lavage continues until the pulmonologist does a visual assessment of the color and sediment of the effluent demonstrating enough clarity to see the movement of fingers through the effluent (Fig. 3). Even 
though Bonella, et al. [10] used optical density monitoring to quantify protein content of the returned fluid as a tool on the decision to terminate the procedure, there is no data that compares optical density values against visual assessment of color and sediment done by an experienced physician.

The length of time to perform OSBSWLL ranged between 135 and $274 \mathrm{~min}$ in our case-series. The duration of single WLL reported by Campo et al. [8] ranged between 120 and $360 \mathrm{~min}$. These results show very similar times between OSBSWLL and single WLL, which could be explained by the fact that the longest part of the procedure is dedicated to positioning the patient and securing/stabilizing the airway, which is the same in both procedures.

The post-procedure management in our cases is similar to other series. Abdelmalak, et al. [11] described an early weaning from the ventilator (within $4 \mathrm{~h}$ of finishing the procedure) and weaning of supplemental oxygen. In our series, $63.3 \%$ of cases were able to be weaned from ventilator and discharged home the same day of the procedure. In fact, $\mathrm{SpO} 2$ values improved by 5.3 points after the procedure; and all patients had a subjective improvement of dyspnea.

Complications reported in other series of WLL have included hypoxemia (which is usually transient, during the emptying of the lung being lavaged, that releases compression of the capillary bed, creating a shunt). Refractory hypoxemia and respiratory failure have been described, mostly related to lavage fluid absorption leading to pulmonary edema [11]. In our series, only 2 cases (6.6\%) required mechanical ventilation longer than $4 \mathrm{~h}$ after OSBSWLL due to hypoxia. One of them, who was treated for pneumonia post-operatively, also had a large residual volume $(2,500 \mathrm{~mL})$ that could have contributed to hypoxia. Pneumothorax has also been described in WLL due to excessive alveolar distention [11]. Even though, none of the patients who underwent OSBSWLL developed pneumothorax, one of the patients who was initially scheduled for OSBSWLL could not complete lavage of the second lung due to hypoxia that was later deemed to be secondary to pneumothorax on the lavaged lung, which required a chest tube placement and admission to ICU. One patient died within 1-year post OSBSWLL, unfortunately no records were able to be collected in terms of cause of death.

We believe that OSBSWLL requires an experienced multidisciplinary team. Planning and communication with the members of the team are the key to the success of this procedure.

Although patients were captured from a prospective, IRB approved database, much of the data was extracted retrospectively. This limited the obtention of some data: clinical presentation, functional outcomes, time of procedure on all patients, overall because most data were recorded before electronic medical records were instituted. Furthermore, despite developing a protocol in our institution, some inconsistency was noted when OSBSWLL started to be performed. Despite all these limitations, we believe that our data can be used to improve future protocols for performing whole lung lavage.

\section{Conclusions}

OSBSWLL can be performed withinone procedure with a same-day discharge as part of the treatment for patients with diagnosis of PAP. OSBSWLL could be considered as part of the management on patients with PAP that require WLL in institutions with a stablished protocol. Standardization of procedural protocols should be developed among institutions.

\section{Acknowledgements}

Not applicable.

\section{Authors' contributions}

J.D.-M.: Conceptualization, Methodology, Investigation, Data curation, WritingReview and Editing, Project administration. E.C.V.: Methodology, Investigation, Data curation, Writing-Original draft. N.P.: Methodology, Data curation, Writing-Original draft. M.S.: Conceptualization, Methodology, Writing-Review and Editing, Project administration. All authors read and approved the final manuscript.

\section{Funding}

No funding was received for this study.

\section{Availability of data and materials}

All data generated and analysed in this study are included in this article under related information files.

\section{Declarations}

\section{Ethics approval and consent to participate}

The Henry Ford Health System Institutional Review Committee (IRB) had approved this study (IRB-9021). Being a retrospective study, the need of informed consent was waived by our IRB.

\section{Consent for publication}

Not applicable.

\section{Competing interests}

All the authors declare NON-COMPETING interests.

\section{Author details}

${ }^{1}$ Interventional Pulmonology, Division of Pulmonary and Critical Care Medicine, Henry Ford Hospital, K-17, 2799 W Grand Blvd, Detroit, Ml 48202, USA. ${ }^{2}$ Department of Medicine, Wayne State University, Detroit, MI, USA. ${ }^{3}$ Pulmonary and Critical Care Medicine, Moffitt Cancer Center, Tampa, FL, USA. ${ }^{4}$ Department of Medicine, University of South Florida, Tampa, FL, USA. ${ }^{5}$ Division of Pulmonary and Critical Care Medicine, University of California Irvine, Irvine, CA, USA.

Received: 23 April 2021 Accepted: 4 November 2021 Published online: 09 November 2021 


\section{References}

1. Gay P, Wallaert B, Nowak S, Yserbyt J, Stavros A, Hermant C, et al. Efficacy of whole-lung lavage in pulmonary alveolar proteinosis: a multicenter international study of GELF. Respiration. 2017;93(3):198-206.

2. Ben-Dov IKY, Rozman J, Soliman A, Bishara H, Zelligson E, Grief J, et al. Pulmonary alveolar proteinosis in Israel: ethnic clustering. Isr Med Assoc J. 1998;1:75-8.

3. Seymour JF, Presneill JJ. Pulmonary alveolar proteinosis: progress in the first 44 years. Am J Respir Crit Care Med. 2002;166:215-35.

4. Wang BM, Stern EJ, Schmidt RA, Pierson DJ. Diagnosing pulmonary alveolar proteinosis. A review and an update. Chest. 1997;111:460-6.

5. Lee KN, Levin DL, Webb WR, Chen D, Storto ML, Golden JA. Pulmonary alveolar proteinosis: high-resolution CT, chest radiographic, and functional correlations. Chest. 1997:111:989-95.

6. Trapnell BC, Whitsett JA, Nakata K. Pulmonary alveolar proteinosis. N Engl J Med. 2003:349:2527-39.

7. Ramirez J, Campbell GD. Pulmonary alveolar proteinosis. endobronchial treatment. Ann Intern Med. 1965:63:429-41.
8. Campo I, Luisetti M, Griese M, Trapnell BC, Bonella F, Grutters JC, et al. WLL international study group a global survey on whole lung lavage in pulmonary alveolar proteinosis. Chest. 2016;150(1):251-3.

9. Stengel PW, Frazer DG, Weber KC. Lung degassing: an evaluation of two methods. J Appl Physiol Respir Environ Exerc Physiol. 1980;48(2):370-5.

10. Bonella F, Bauer PC, Griese M, Wessendorf TE, Guzman J, Costabel U. Wash-out kinetics and efficacy of a modified lavage technique for alveolar proteinosis. Eur Respir J. 2012;40:1468-74.

11. Abdelmalak BB, Khanna AK, Culver DA, Popovich MJ. Therapeutic whole lung lavage for pulmonary alveolar proteinosis: a procedural update. J Bronchology Interv Pulmonol. 2015;22(3):251-8.

\section{Publisher's Note}

Springer Nature remains neutral with regard to jurisdictional claims in published maps and institutional affiliations.
Ready to submit your research? Choose BMC and benefit from:

- fast, convenient online submission

- thorough peer review by experienced researchers in your field

- rapid publication on acceptance

- support for research data, including large and complex data types

- gold Open Access which fosters wider collaboration and increased citations

- maximum visibility for your research: over $100 \mathrm{M}$ website views per year

At BMC, research is always in progress.

Learn more biomedcentral.com/submissions 